\title{
The vibrational properties of xenon hydrate: An inelastic incoherent neutron scattering study
}

\author{
Christian Gutt, ${ }^{\text {a) }}$ Julian Baumert, and Werner Press \\ Institut für Experimentelle und Angewandte Physik der Universität Kiel, Kiel, Germany \\ John S. Tse \\ National Research Council of Canada, Ottawa, Ontario, Canada \\ Stefan Janssen \\ Laboratory for Neutron Scattering, Paul Scherrer Institut, Villigen, Switzerland
}

(Received 11 September 2001; accepted 5 December 2001)

\begin{abstract}
We report results from a high-resolution, incoherent inelastic neutron scattering (IINS) study of xenon hydrate. This study extends previous work in which the existence of a strong coupling between localized guest vibrations and the lattice modes was shown ${ }^{1}$ for the first time [Tse et al., Europhys. Lett., 54, 354 (2001)]. This guest-host coupling might be responsible for the glass-like temperature dependence of the thermal conductivity of the crystalline gas hydrates. Our experiment focused on the low-frequency phonon density of states of the ice-like water lattice of xenon hydrate. We found two broad maxima in the density of states (DOS) at energy transfers of 7.3 and $10.3 \mathrm{meV}$. The first peak is assigned to the transverse acoustic (TA) phonons near the zone boundary and the second to the fold-back of the TA modes towards the zone center. The guest-host coupling could be confirmed by finding three distinct low energy peaks in the DOS at energy transfers of 2.05, 2.87, and $3.94 \mathrm{meV}$. In addition, another broad inelastic scattering component extending from 0 to 1.5 $\mathrm{meV}$ has been found, which may also be important for the low-temperature behavior of the thermal conductivity. The line positions of the coupled modes shift to higher frequencies with increasing temperature, pointing towards the importance of the repulsive part of the host-guest interaction which is responsible for the stability of gas hydrate structures. (C) 2002 American Institute of Physics. [DOI: 10.1063/1.1446426]
\end{abstract}

\section{INTRODUCTION}

Clathrates are inclusion compounds in which small guest molecules or atoms are trapped in cages formed by a host network. Recently, two isostructural clathrates attracted considerable interest for quite different reasons. On the one hand there are the gas hydrates, in which hydrophobic guests, e.g., methane or xenon, are trapped in cages formed by an ice-like hydrogen-bonded network of water molecules. The interest in the gas hydrates results from large accumulations of natural hydrates (mostly methane hydrate) on the oceanic seafloors, where they have a major impact on the stability of continental margins. ${ }^{1}$ The methane gas stored in these deposits may serve as a future energy supply, released methane from these deposits may contribute to the greenhouse effect. Therefore, physical properties of methane hydrates, e.g., the low thermal conductivity, also are of great interest.

On the other hand there are the semiconductor clathrates, in which small metal atoms, e.g., $\mathrm{Na}$ or $\mathrm{Ba}$, are trapped in cages formed by a covalently bonded network of elements, e.g., Si or Ge. The clathrate structures can be understood as a close packing of polyhedral cages, each containing one guest atom or molecule. In this paper we will concentrate on the structure type I (space group Pm3n, $a_{0} \sim 12 \AA$ ) of the clathrate family, which consists of eight cages per unit cell: two

${ }^{\text {a)} E l e c t r o n i c ~ m a i l: ~ g u t t @ p h y s i k . u n i-d o r t m u n d . d e ~}$ small dodecahedral cages and six large tetrakaidecahedral cages.

The semiconductor clathrates attract much interest because they may provide a new class of thermoelectric materials due to their thermal and electronic properties (see, e.g., Refs. 2-5). The figure of merit of thermoelectric materials is the dimensionless quantity $Z T$, where $T$ is the temperature and $Z=S^{2} \sigma / \kappa . S$ is the Seebeck coefficient, $\sigma$ is the electri$\mathrm{cal}$, and $\kappa$ is the thermal conductivity. Therefore, good thermoelectric materials should provide both a high electronic conductivity and a low thermal conductivity. ${ }^{6}$ Slack $^{7,8}$ called such a material a phonon glass and an electron single crystal.

Both materials, the gas hydrates as well as the semiconducting clathrates, display an unusually low thermal conductivity in comparison with crystals solely composed of the cage-forming elements, i.e., ice Ih and, fcc Si or Ge. Even more surprising is the temperature behavior of the thermal conductivity, which is similar to that of glasses despite the crystalline character of the clathrates. ${ }^{9}$

There are at least two possible reasons for this behavior of the thermal conductivity: (i) the large unit cell in combination with the open framework structure and (ii) a coupling between guest and host vibrations in the low-frequency region. Considering the large unit cell and the open framework structure, we may follow the arguments given by Slack, ${ }^{10}$ who stated that the thermal conductivity $\kappa$ scales with the number of atoms $n$ in the unit cell as $\kappa \sim 1 / n^{2 / 3}$ (see also 
Dong et al. ${ }^{11}$ ). Due to the large size of the unit cells this scaling could reduce the thermal conductivity $\kappa$ of the clathrates considerably.

In addition to the large size of the unit cell and the open framework structure, there are also encaged guest atoms or molecules interacting with the surrounding cage structure. This guest-host interaction is weaker than the bond strength of the cage structure but its importance may be seen particularly from the gas hydrates in which the encaged guests stabilize the ice framework via the repulsive interaction, i.e., an empty hydrate lattice is not stable. It is this guest-host interaction which is thought to provide an effective phonon scattering mechanism leading to a further reduced thermal conductivity and to the glass-like temperature behavior of the clathrates: the encaged guests perform low-frequency localized rattling modes in the cages acting via the guest-host coupling as possible scattering centers for the heat-carrying acoustic lattice phonons. The existence of such a coupling was first found in MD simulations ${ }^{12,13}$ and recently was confirmed by means of an experiment with inelastic neutron scattering (IINS) of xenon hydrate $\left(8 \mathrm{Xe} \cdot 46 \mathrm{H}_{2} \mathrm{O}\right) .{ }^{14}$ Due to the importance of this finding, not only for the gas hydrates but also for the semiconducting clathrates, we studied the vibrational dynamics of xenon hydrate in more detail (indirect hints for a guest-host coupling in Si-clathrates have been found by Melinon et al. ${ }^{15}$ in IINS experiments). Xenon hydrate is especially well suited for the investigation of guest-host coupling with IINS for reasons of scattering intensity. The scattering signal of the $\mathrm{H}_{2} \mathrm{O}$ network exceeds that of the xenon atoms by nearly three orders of magnitude. Thus, the IINS signal displays the vibrational properties of the host lattice only. The thermal conductivity of xenon hydrate has been measured by Handa, ${ }^{16}$ who found low values similar to those reported for other gas hydrate pointing towards universal behavior of the thermal conductivity of these compounds.

In this paper we report on an IINS experiment on xenon hydrate with high energy resolution of $100 \mu \mathrm{eV}$ FWHM in the temperature regime from 50 to $180 \mathrm{~K}$. The aim of the experiment was to investigate the guest-host coupling in detail and to measure the phonon density of states of xenon hydrate.

The paper is organized as follows: in Sec. II we present the experimental details of our study. The results of the IINS experiment of xenon hydrate are presented and discussed in Sec. III. Section IV contains the summary and conclusions.

\section{EXPERIMENTAL DETAILS}

Clathrate hydrates formed by small guest molecules or atoms like xenon destabilize at atmospheric pressure when the temperature is raised above $200 \mathrm{~K}$. The synthesis of these clathrates requires a high gas pressure of the guest molecules. After removing the pressure it is necessary to handle and store the sample at temperatures well below $200 \mathrm{~K}$. This can best be achieved under conditions of liquid nitrogen temperature. The sample has been prepared at the NRC, Canada from $\mathrm{Xe}$ and $\mathrm{H}_{2} \mathrm{O}$ in a process described in detail elsewhere. ${ }^{17}$ It was shipped to Kiel in a liquid $\mathrm{N}_{2}$ dewar. With exactly the same process we prepared a deuterated
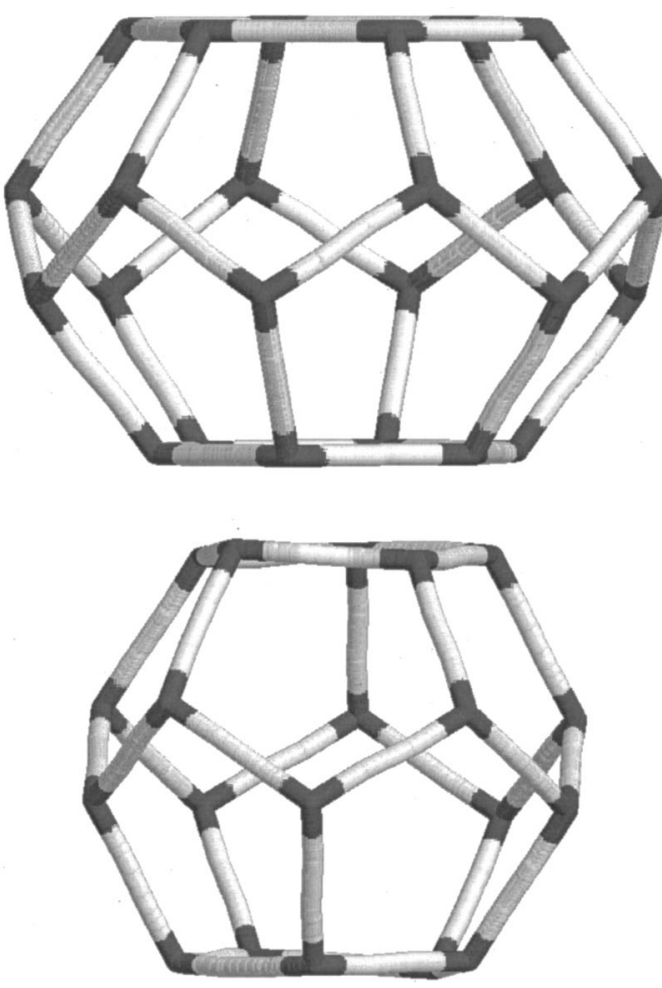

FIG. 1. The two types of cages present in the structure type I of xenon hydrate. The ellipsoidal shape of the large cage (top) leads to the two lowenergy coupled modes at 2.05 and $2.87 \mathrm{meV}$, while the spherical shape of the small cage (bottom) leads to the coupled mode at $3.94 \mathrm{meV}$ (see Fig. 2).

sample, i.e., $\mathrm{Xe} \cdot \mathrm{D}_{2} \mathrm{O}$, which has been studied by a highresolution neutron diffraction experiment at the diffractometer E9 at the HMI, Berlin. ${ }^{18}$ The cubic hydrate structure I was found at temperatures of 5 and $150 \mathrm{~K}$, with lattice constants of $a_{0}=11.821(1) \AA$ and $a_{0}=11.869(1) \AA$, respectively (see also Ref. 19). The structure consists of eight cages per unit cell: six large cages and two small cages (Fig. 1). Rietveld analysis shows ${ }^{18}$ that all large cages are fully occupied by one xenon atom while only $80 \%$ of the small cages are occupied, leading to the chemical formula 7.6 Xe. $46 \mathrm{H}_{2} \mathrm{O}$. For the IINS experiment, the fine hydrate powder was mounted into an aluminum sample cell of $1 \mathrm{~mm}$ thickness. After this procedure the sample was cooled down to $50 \mathrm{~K}$ in a liquid helium cryostat. The temperature was controlled by a $\mathrm{Rh}-\mathrm{Fe}$ sensor fixed to the sample stick. Inelastic measurements have been performed on the new timeof-flight spectrometer FOCUS ${ }^{20}$ at the Paul-Scherrer Institute, Switzerland in its time-focusing mode. An initial neutron wavelength of $\lambda=5.0 \AA$ was selected by the 002 reflection of a pyrolytic graphite monochromator. The use of the time-focusing option of the spectrometer resulted in a good energy resolution of about $0.1-0.3 \mathrm{meV}$ (FWHM) in the region of neutron energy gain of $0-10 \mathrm{meV}$, yielding well-defined spectra. The resolution function and detector efficiency have been measured by means of a vanadium standard. The signal of the empty aluminum sample cell and a flat background have been subtracted. IINS spectra have been recorded at temperatures of 50,100, 150, and $180 \mathrm{~K}$, respectively. 


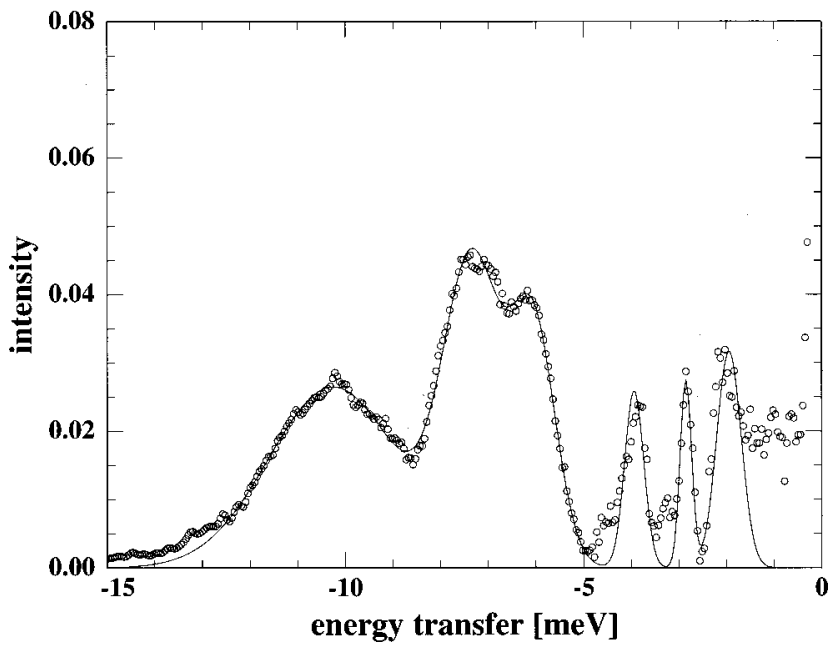

FIG. 2. Inelastic neutron scattering spectrum of xenon hydrate (7.6 Xe . $46 \mathrm{H}_{2} \mathrm{O}$ ) at $T=100 \mathrm{~K}$ and $\lambda=5 \AA$. Spectrum measured at the neutron time-of-flight spectrometer FOCUS, PSI Switzerland. The two broad peaks at energy transfers of 7.3 and $10.3 \mathrm{meV}$ are assigned to the (TA) phonons near the zone boundary and the fold-back of the TA modes towards the zone center. The three distinct peaks at low-energy transfers of 2.05, 2.87, and $3.94 \mathrm{meV}$ arise from a coupling between the vibrations of the localized xenon atoms in the cages and the surrounding ice-like water lattice. In addition, there exists a broader contribution to the inelastic scattered signal in the energy region between 1.5 to $0 \mathrm{meV}$.

\section{RESULTS AND DISCUSSION}

Figure 2 shows a typical spectrum of xenon hydrate at $T=100 \mathrm{~K}$ in the region of energy transfers from 15 to $0 \mathrm{meV}$ (measurement in neutron energy gain, that is deexcitation of phonons, limits experiments to $T>50 \mathrm{~K}$ ). For intensity reasons the signal from all detectors in the accessible Q-range from $0.4-3.2 \AA^{-1}$ has been summed up. Five distinct peaks are the most prominent features of the spectrum. They have been found at all measured temperatures. Two peaks are relatively broad and are at positions of 7.3 and $10.3 \mathrm{meV}$, while there is also a weaker feature at $5.9 \mathrm{meV}$. The fit with Gaussians leads to HWHMs of $0.8 \mathrm{meV}(0.4 \mathrm{meV}$ for the peak at $5.9 \mathrm{meV}$ ) and $1.4 \mathrm{meV}$, respectively. The first maximum at $7.3 \mathrm{meV}$ is assigned to the transverse acoustic (TA) phonons near the zone boundary, and the secondary peak can be attributed to a high phonon density of states arising from the fold-back of the transverse acoustic phonon modes towards the center of the Brillouin zone. ${ }^{14}$ Of particular interest are the three sharp lines appearing at small energy transfers of $2.05,2.87$, and $3.94 \mathrm{meV}( \pm 0.02 \mathrm{meV})$. An analysis of the line positions as a function of $\mathrm{Q}$ showed no dispersion and the corresponding linewidths (FWHM) provide small values of $0.2,0.13$, and $0.31 \mathrm{meV}$, respectively, indicating an optic mode character of these excitations. These lines are absent in the known ice spectra, see, e.g., Ref. 21, and indirectly show the coupling between guest and host lattice vibrations in the gas hydrate systems: ${ }^{14}$ In the case of xenon hydrate the ratio of $6.05 \mathrm{H}_{2} \mathrm{O}$ molecules to one xenon atom leads to a ratio of the incoherent scattering cross section of nearly 1000:1. Besides the incoherent scattering cross section, the amplitudes of the vibrational motions are important for the observed intensity. However, from neutron diffraction experiments, ${ }^{19,18}$ it is known that the vibrational amplitudes of the encaged xenon atoms are only slightly smaller than those of the $\mathrm{H}$ atoms. Therefore, it is clear that the measured inelastic signal displays the density of states of the $\mathrm{H}$ atoms only. As this low-frequency region is dominated by the translational vibrations, the measured spectrum represents the DOS of the water molecules.

In calculations of the hydrate lattice dynamics ${ }^{12}$ it has been found that the xenon atoms in the cages behave like Einstein oscillators, providing three different vibrational frequencies: The xenon atoms in the small dodecahedral cages (Fig. 1) are calculated to vibrate with the highest frequency of $4.4 \mathrm{meV}$, while the ellipsoidal shape of the large cage leads to two lower vibrational frequencies of 2.2 and 3.2 $\mathrm{meV}$, respectively. The coupling of the xenon atoms to the surrounding ice cages causes cage oscillations with the same frequency, making them visible in the IINS spectrum. From a lattice dynamical point of view the peaks in the host lattice density of states arise from a symmetry avoided crossing between the flat xenon branches and the acoustic host lattice vibrations of the same symmetry. Factor group analysis ${ }^{22}$ showed that both guest and host vibrations contain the same $T_{1 u}$ symmetry term, resulting in an avoiding of the crossing. The resulting flat host lattice branches provide a high density of states which shows up in the sharp lines in the neutron spectrum.

Regarding the relative intensities, a simplistic (single particle) consideration yields an intensity ratio of about 2:1:1 (three lines, order of increasing energy) as there are six large and two small cages in the unit cell. However, we found a ratio close to $2: 1: 2$ pointing towards a stronger guest-host coupling in the small cages which has also been found in MD simulations of xenon hydrate. ${ }^{23}$

From the spectrum in Fig. 2 it becomes clear that our simple Gaussian ansatz does not fit the line shapes of the three peaks very well. The lines at 2.87 and $3.94 \mathrm{meV}$ are accompanied by weaker features at energy transfers of 3.2 and $4.8 \mathrm{meV}$. On the other hand, the line at $2.05 \mathrm{meV}$ is accompanied by a broad distribution of inelastic or quasielastic excitations extending from the elastic line to energy transfers of $1.5 \mathrm{meV}$. The latter could be especially important for the low-temperature behavior of the thermal conductivity; for $\mathrm{Sr}_{8} \mathrm{Ga}_{16} \mathrm{Ge}_{30}$-clathrate, Cohn et al. found a conductivity with the glass-like behavior down to temperatures of $0.1 \mathrm{~K}^{5}$ As the translational vibrations of the guests are frozen in at such low temperatures, an explanation based on static disorder of the guests in the cages has been proposed. Small energies $(<1 \mathrm{~K})$ characterize the separation between these guest positions in the cages and give rise to tunneling states like in the glasses (see, e.g., Ref. 24). However, neutron diffraction experiments of xenon hydrate do not lead to any evidence for an exceptionally large static disorder of the xenon atoms in either type of cage at low temperatures. ${ }^{18,19}$ Thus, if there exists such a distribution of stable positions around the cage centers, they must be arranged quite close to the cage center. In the case of gas hydrates the situation at low temperatures is even more complicated due to the proton disorder of the ice-like host lattice which leads to a broad distribution of local potentials in the cages. ${ }^{25}$ One may speculate whether this complex equipotential surface at the 

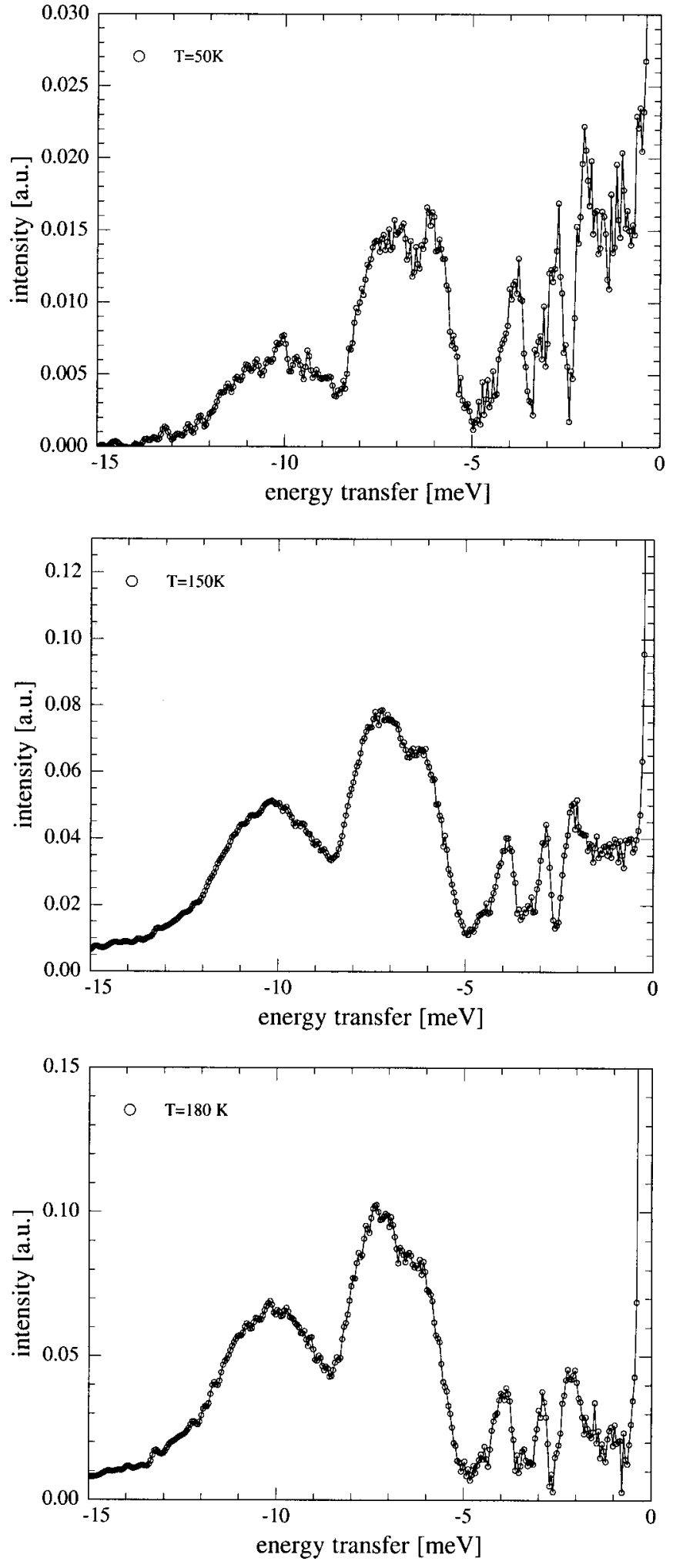

FIG. 3. IINS spectra at temperatures of 50, 150, and $180 \mathrm{~K}$, respectively.

cage centers gives rise to the broad distribution of energy transfers in the low-energy region and thus may be responsible for the low-temperature thermal conductivity. Also, the disorder due to partly empty small cages could reduce the thermal conductivity.

Figure 3 displays the IINS spectra of xenon hydrate at temperatures of 50,150, and $180 \mathrm{~K}$. All spectra show the same excitations with the different line intensities mainly arising from the Boltzmann factor. However, a comparison of the spectra also suggests the existence of a broad quasielastic component beneath the inelastic excitations. There is no explanation for this scattering component yet.

The temperature dependence of the line positions is of special interest. When raising the temperature from 50 to 180 $\mathrm{K}$ we observed a small increase in all three coupled mode frequencies by about $\Delta E \sim 100 \mu \mathrm{eV}$. A similar small increase of $\Delta E \sim 100-200 \mu \mathrm{eV}$ has been found in MD simulations of Inoue $\mathrm{et} \mathrm{al.}^{26}$ This increase in frequency with increasing temperatures indicates that anharmonic terms play a major role in the guest-host interaction of the hydrate systems. Due to the large vibrational amplitudes of the encaged guests, the anharmonic terms originate most probably from the repulsive part of the guest-host interaction. The situation is similar to that in the lattice dynamics of rare gas solids. ${ }^{27}$ The influence of anharmonic terms on the frequencies of the lattice dynamics has been treated by Hooton ${ }^{28}$ in perturbation theory

$$
\omega^{\prime 2}=\omega^{2}+\frac{U^{4}(x)}{2 \sqrt{m_{1} m_{2}}}\left\langle x^{2}\right\rangle,
$$

where $\left\langle x^{2}\right\rangle$ denotes the vibrational amplitude, $U^{4}(x)$ $=\partial^{4} U / \partial x^{4}$ with the interaction potential $U$, and $m_{1}$ and $m_{2}$ the masses of the water molecule and xenon atom, respectively. In the case of a dominating repulsive interaction we find $U^{4}(x)>0$ and the vibrational frequencies increase with increasing vibrational amplitudes of the guest atoms, i.e., with increasing temperature.

The question why the relatively weak van der Waals forces between guest and host strongly influence the lattice dynamics may be understood on the basis of a simple phenomenological model (for a more elaborated treatment see, e.g., Ref. 29). Systems in which dispersive long wavelength modes interact with localized oscillators are well known in solid-state physics. The behavior in xenon hydrate closely parallels the behavior of electromagnetic waves propagating through a medium containing localized ions resulting in the polariton ${ }^{30}$ or the behavior of sound waves traveling through a medium containing resonant spins. ${ }^{31}$ In our case we assume an elastic continuum, i.e., sound waves representing the host lattice phonons interacting with the displacement field $h$ of localized guest oscillators. The uncoupled elastic host field $u$ of density $\rho$ and sound velocity $v$ can be described by the Hamiltonian $H_{1}$

$$
H_{1}=\frac{p_{1}^{2}}{2 \rho}+v^{2} k^{2} u^{2} .
$$

The Hamiltonian $\mathrm{H}_{2}$ of the encaged xenon atoms is that of a harmonic oscillator with frequency $\omega_{0}$ and displacement $h$

$$
H_{2}=\frac{p_{2}^{2}}{2 m}+m \omega_{0}^{2} h^{2} .
$$

We assume a simple bilinear coupling term $u \cdot h$ of the two displacement fields $u$ and $h$, leading to the coupled Hamiltonian

$$
H_{C}=H_{1}+H_{2}+\alpha u h,
$$




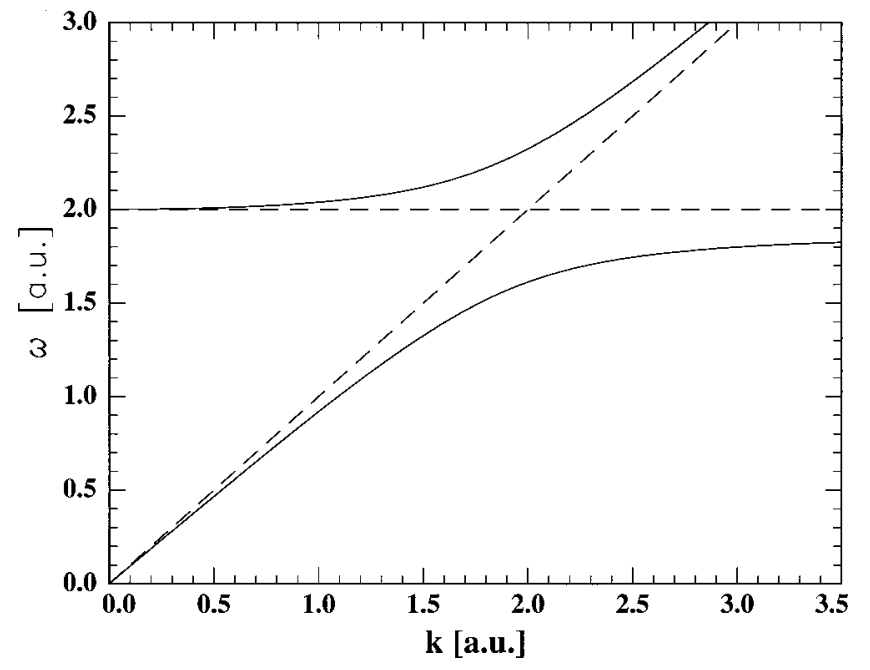

FIG. 4. Dispersion relation as deduced from our simple phenomenological model [Eq. (5)]. The dashed lines represent the uncoupled modes, i.e., the linear acoustic lattice modes and the Einstein frequency of the localized guest vibration in the hydrate cages. The solid lines represents the coupled modes in which an interaction between both modes is taken into account. At the resonance frequency $\omega_{0}$ of the encaged guests the interaction between host and guest is strong, resulting in a change of the host lattice modes. The flat part of this lattice mode shows up in the IINS spectra.

with coupling strength $\alpha$. We want to note that the coupling strength $\alpha$ may be considerably larger than expected for weak van der Waals forces as the rattling xenon atoms probe the strongly repulsive part of the interaction potential. A solution of the classical equations of motions can be found by assuming a wave-like behavior of $u$ and $h$ as $e^{(i(\omega t-k x))}$ resulting in the dispersion relation

$$
k^{2} v^{2}=\omega^{2}+\frac{\alpha^{2} \rho^{\prime} \omega^{2}}{m\left(\omega_{0}^{2}-\omega^{2}\right)},
$$

which is displayed in Fig. 4. The dispersion relation is in qualitative agreement with dispersion curves found in MD and LD calculations of gas hydrates and Ge-clathrates. From the figure it becomes clear that the long wavelength host lattice phonons are indeed strongly affected by the guesthost coupling. At the crossing of guest and host vibrations, the acoustic host phonons branches bend to flat dispersion branches with corresponding high density of states. In the case of resonance the host lattice waves become standing waves, that is, they are reflected by the guest atoms. From Eq. (5) we see that the weak coupling constant $\alpha$ of the van der Waals forces in the gas hydrate systems is counterbalanced by the resonance effect as $\omega \rightarrow \omega_{0}$, leading to a strong effect on the dispersion relation of the host lattice.

\section{CONCLUSION}

We performed a high-resolution IINS experiment on xenon hydrate from which we obtained detailed information about the vibrational properties of xenon hydrate. The existence of a guest-host coupling has been confirmed by the observation of three sharp peaks at energy transfers of 2.05, 2.87 , and $3.94 \mathrm{meV}( \pm 0.02 \mathrm{meV})$ at $T=100 \mathrm{~K}$. These peaks are attributed to resonant cage vibrations of the ice-like host lattice at the frequencies of the encaged xenon atoms. In addition, we found a broader distribution of energy transfers extending from 0 to $1.5 \mathrm{meV}$, the origin of which is unknown. The temperature dependence of the coupled modes has been measured. With increasing temperature we found the coupled modes to increase in frequency, pointing towards the importance of the repulsive interaction between the xenon atoms and the host lattice. It is this repulsive interaction which stabilizes the gas hydrate structures. The experiment also shows the need for a complete lattice dynamical calculation, which is no simple task, due to the large unit cell, the proton disorder, and the anharmonicity of the guest-host interaction in xenon hydrate.

${ }^{1}$ E. D. Sloan, Clathrates Hydrates of Natural Gases (Dekker, New York, 1990).

${ }^{2}$ N. P. Blake, L. Mollnitz, G. Kresse, and H. Metiu, J. Chem. Phys. 111, 3133 (1999)

${ }^{3}$ G. S. Nolas, J. L. Cohn, G. A. Slack, and S. B. Schujman, Appl. Phys. Lett. 73, 178 (1999).

${ }^{4}$ G. S. Nolas, T. J. R. Weakley, J. L. Cohn, and R. Sharma, Phys. Rev. B 61, 3845 (2000)

${ }^{5}$ J. L. Cohn, G. S. Nolas, V. Fessatidis, T. H. Metcalf, and G. A. Slack, Phys. Rev. Lett. 82, 779 (1999).

${ }^{6}$ J. S. Tse, K. Uehara, R. Rousseau, A. Ker, C. I. Ratcliffe, M. A. White, and G. MacKay, Phys. Rev. Lett. 85, 114 (2000).

${ }^{7}$ G. Slack, in CRC Handbook of Thermoelectrics, edited by D. M. Rowe (CRC, Boca Raton, 1995).

${ }^{8}$ G. Slack, Mater. Res. Soc. Symp. Proc. 478, 47 (1997).

${ }^{9}$ R. G. Ross, P. Andersson, and G. Bäckström, Nature (London) 290, 322 (1981).

${ }^{10}$ G. A. Slack, in Solid State Physics (Academic, New York, 1979), Vol. 34, p. 1.

${ }^{11}$ J. Dong, O. F. Sankey, and C. W. Myles, Phys. Rev. Lett. 86, 2361 (2001).

${ }^{12}$ J. S. Tse, M. L. Klein, and I. R. McDonald, J. Chem. Phys. 78, 2096 (1983).

${ }^{13}$ J. S. Tse, M. L. Klein, and I. R. McDonald, J. Chem. Phys. 12, 6146 (1984).

${ }^{14}$ J. S. Tse, V. P. Shpakov, V. R. Belosludov, F. Trouw, Y. P. Handa, and W. Press, Europhys. Lett. 54, 354 (2001).

${ }^{15}$ P. Melinon, P. Keghelian, A. Perez, B. Champagnon, Y. Guyot, L. Saviot, E. Reny, C. Cros, M. Pouchard, and A. J. Dianoux, Phys. Rev. B 59, 10099 (1999).

${ }^{16}$ Y. P. Handa and J. G. Cook, J. Phys. Chem. 91, 6327 (1987).

${ }^{17}$ Y. P. Handa, Ind. Eng. Chem. Res. 27, 872 (1988).

${ }^{18}$ C. Gutt, W. Press, J. S. Tse, and D. Többens (unpublished).

${ }^{19}$ T. Ikeda, S. Mae, O. Yamamuro, T. Matsuo, S. Ikeda, and R. M. Ibberson, J. Phys. Chem. A 104, 10623 (2000).

${ }^{20}$ J. Mesot, S. Janssen, L. Holitzner, and R. Hempelmann, J. Neutron Res. 3, 293 (1996).

${ }^{21}$ J. C. Li, J. Chem. Phys. 105, 6733 (1996).

${ }^{22}$ J. S. Tse, C. I. Ratcliffe, B. M. Powell, V. F. Sears, and Y. P. Handa, J. Phys. Chem. 101, 4491 (1997).

${ }^{23}$ J. S. Tse, B. M. Powell, V. J. Sears, and Y. P. Handa, Chem. Phys. Lett. 215, 383 (1993)

${ }^{24}$ U. Buchenau, Y. M. Galperin, V. L. Gurevich, and H. R. Schober, Phys. Rev. B 43, 5039 (1991).

${ }^{25}$ C. Gutt, W. Press, A. Hüller, J. S. Tse, and H. Casalta, J. Chem. Phys. 114, 4160 (2001).

${ }^{26}$ R. Inoue, H. Tanaka, and K. Nakanishi, J. Chem. Phys. 104, 9569 (1996).

${ }^{27}$ N. R. Werthamer in Rare Gas Solids, edited by M. L. Klein and J. A. Venables (Academic, London, 1976), Vol. 1.

${ }^{28}$ D. J. Hooton, Philos. Mag. 46, 422 (1955).

${ }^{29}$ J. S. Tse, J. Inclusion Phenom. Mol. Recognit. Chem. 17, 259 (1994).

${ }^{30}$ N. W. Ashcroft and N. D. Mermin, Solid State Physics (Saunders College, Philadelphia, 1976).

${ }^{31}$ E. H. Jacobsen and K. W. H. Stevens, Phys. Rev. 129, 2036 (1963). 